\title{
Enhancement of images corrupted with signal dependent noise: Application to ultrasonic imaging
}

\author{
M. Alper Kutay, Mustafa Karaman, Gözde Bozdağg 1 \\ Department of Electrical and Electronics Engineering \\ Bilkent University, Ankara, 06533 TURKEY
}

\begin{abstract}
An adaptive filter for smoothing images corrupted by signal dependent noise is presented. The filter is mainly developed for speckle suppression in medical B-scan ultrasonic imaging. The filter is based on mean filtering of the image using appropriately shaped and sized local kernels. Each filtering kernel, fitting to the local homogeneous region, is obtained through local statistics based region growing. Performance of the proposed scheme have been lested on a B-scan image of a standard tissue-mimicking ultrasound resolution phantom. The results indicate that the filter effectively reduces the speckle while preserving the resolvable details. The performance figures obtained through computer simulations on the phantom image are presented in a comparative way with some existing speckle suppression schemes.
\end{abstract}

\section{INTRODUCTION}

Noise filtering is one of the important issues in image processing. In general, images can be corrupted by sigıal dependent (multiplicative) and signal independent (additive) noise. Linear filtering techniques perform well in climination of additive noise whereas they are not efficient for filtering signal dependent noise. Suppression of signal dependent noise involves adaptive and/or non-linear filtering techniques such as adaptive non-linear mean filtering $[1,2]$ and local statistics based filtering $[3,4]$.

Because of coherent nature of phased array medical ultrasound imaging scanners, constructive and destructive interference effects of backscattered echoes from unresolvable random tissue inhomogeneities results in mottled B-scan inages $[5,6,7]$. . This occurs especially in imaging organs such as liver and kidney whose underlying structures are 1.00 small to be resolved by ultrasound scanners. Speckle is the term used for granular pattern appeared on B-scans due to the mottling, and considered as a kind of signal dependent noise. Speckle degrades image quality of B-scans, and hence reduces the ability of human observer for discrimination of fine details in diagnostic examination. It also limits potential of further image processing such as edge detection and flow imaging. To overcome these problems, suppression of speckle has been subject of considerable research. 
Ultrasound B-scan images have some special features which has to be preserved by the filtering, such as bright large scale interfaces between organs, structures with dimensions comparable to speckle size, boundaries between two regions with slightly different gray levels. Therefore, linear non-adaptive smoothing techniques used for other image processing purposes may not be adequate for ultrasound images. Many of such techniques introduce severe blurring and/or show unacceptable performance in elimination of speckle. Various non-linear and adaptive techniques have been proposed in the literature. Most of these techniques involve non-linearity and speckle statistics, and change filter parameters, such as kernel size and weighting, locally according to local statistics and image content $[3,8,9,10,11,12,13,14,15]$.

Identification of characteristic parameters of speckle statistics has a critical importance for speckle suppression i.echniques based on adaptive post-filtering. It has been shown that the envelope detected speckle echo signal has Rayleigh distribution with mean proportional to standard deviation [5]. On the other hand, Loupas et. al. [11] has shown that non-linear processing (such as logarithmic compression) employed on ultrasound echo images affect the speckle statistics, and so the local mean becomes proportional to the local variance rather than the standard deviation. That is, the ratio of variance to mean for fully developed speckle is constant. This result have been used in adaptation of filter parameters to smooth out speckle from B-scans $[11,12,14,15]$. In this paper, we present our results in a comparative way with two speckle suppression methods proposed in [11, 12].

In this study, an adaptive speckle suppression filter (ASSF) based on smoothing images corrupted by signal dependent noise is presented. The filter adaptation is achieved by using appropriately shaped and sized local filtering kernels where each kernel effectively fits to an arbitrary shaped homogeneous region containing the processed pixel since no limitation on kernel shape is imposed. Each kernel is obtained through region growing technique employing image local statistics as the region growing criteria. Performance of the technique is tested on a B-scan image of a $\therefore$ tandard tissue-mimicking ultrasound resolution phantom.

In section II, we discuss how the local statistics of ultrasound images are used as a growing criteria. The effect of the filtering parameters to the final performance is also described in section II. In section III results of the filter is illustrated and comparisons with other methods are presented.

\section{THE FILTER}

To smooth out speckle from a B-scan image, the first step is to identify regions consisting of speckle pattern and resolvable structures. Ratio of local variance to mean for fully developed speckle, which is constant, can be easily measured on a B-scan image. Then, using the measured local statistics, it is possible to decide whether the processed pixel location within a speckle region or not. In general, if the local statistics is larger than that of fully developed speckle, then the local pixel can be considered as a point in a resolvable structure. Otherwise it belongs to a region consisting of speckle pattern, and hence is subject to smoothing. It should be, however, noted that such a hard statistical bound used for the decision may cause undesired results. On the other hand, shape of speckle pattern and average speckle size vary at different locations of sector images. Consequently, each homogeneous region used for smoothing may have an arbitrary shape and size. The designed ASSF handles above limitations in speckle smoothing. This is achieved through a region growing procedure which effectively fits the grown region to the homogeneous area without imposing any shape constraint. The growing procedure employs a look-up table consisting of statistical bounds for different values of local statistics, instead of using a single hard bound as the growing criteria. Overall procedure of ASSF can be described in three main steps: computation of local statistics, region growing, and averaging (or median) operation.

To choose a proper window size, which precisely approximates the statistics, local mean and variance on fully 


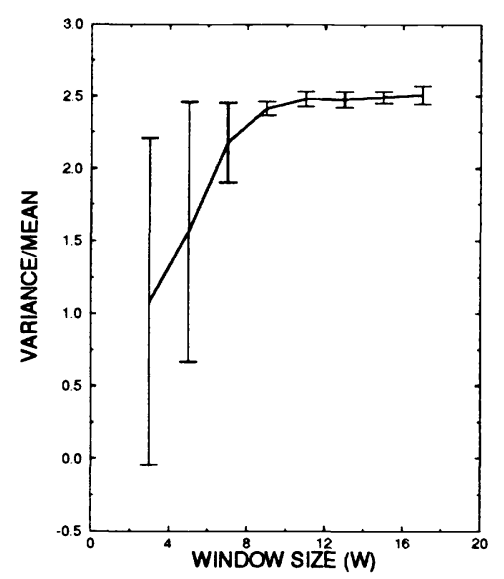

Fig. 1: The ratio of variance to mean vs window size.

developed speckle areas are measured for different sized square windows. The following expressions are used in these measurements:

$$
\begin{gathered}
\mu_{i, j}=\frac{1}{W^{2}} \sum_{m=1}^{W} \sum_{n=1}^{W} x_{i-m, j-n} \\
\sigma_{i, j}^{2}=\frac{1}{W^{2}} \sum_{m=1}^{W} \sum_{n=1}^{W}\left(x_{i-m, j-n}-\mu_{i, j}\right)^{2}
\end{gathered}
$$

where $x_{i, j}$ is the pixel at the location $(i, j)$, and $W \times W$ is the size of square window. For the sake of simplicity, a single parameter representing the local statistics is defined as:

$$
\alpha_{i, j}=\sigma_{i, j}^{2} / \mu_{i, j}
$$

The measurements are depicted in Fig. 1 as a plot of ratio of variance to mean vs window size. For each window size, the variance and mean computations are carried out at different locations on fully developed speckle, and the results are averaged. The figure indicates that window size larger than $11 \times 11$ can precisely approximates the asymptotic value of speckle statistics. Based on this observation, the window size is chosen as $W=11$ for computations of local statistics in the following measurements and also in the filtering. Note that this window size may not be suitable for other ultrasound scanners, and hence it must be measured prior to the filtering. Also, the size must be kept sufficiently small for accurate detection of resolvable structures.

Region growing is a procedure used to form groups of pixels satisfying a certain homogeneity criteria $[16,17,18]$. Depending on the application, the homogeneity criteria, which is the critical point in growing, can be a function of various image parameters to characterize homogeneity from different point of view, such as gray level, texture, local statistics, and color similarities.

In ASSF, the local statistics, ratio of local variance to local mean, is used as the quantitative measure in obtaining a homogeneous region to be used as the filtering kernel for each image pixel. To do this, a fixed sized square window, which precisely approximates the statistics of the fully developed speckle, is chosen to compute the local statistics for all pixels. In region growing, each image pixel is taken as a seed point. Then, any pixel with local statistics close io the seed pixel's local statistics within a certain bound, is included to the region of the seed pixel. This search is repeated for all pixels which are spatially close to the seed pixel within a certain distance bound. In other words, to test whether a pixel $(m, n)$ belongs to the homogeneous region of a seed pixel $(i, j)$, the following must be satisfied:

$$
\alpha_{m, n}-\Delta \alpha\left(\alpha_{i, j}\right)<\alpha_{m, n} \leq \alpha_{m, n}+\Delta \alpha\left(\alpha_{i, j}\right)
$$




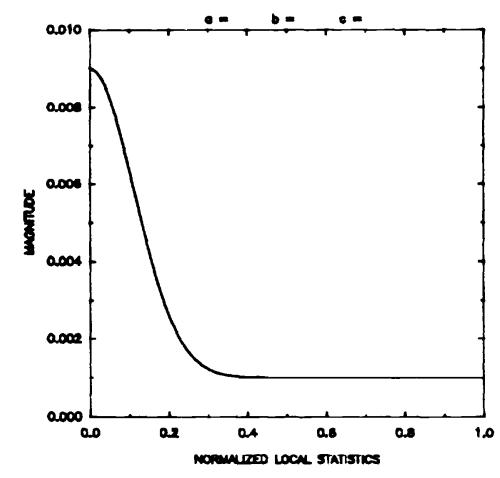

Fig. 2: The statistical similarity bound, $\Delta \alpha($.$) as a function of normalized \alpha_{i, j}$.

$$
\begin{aligned}
& \text { Pixel }(m, n) \text { is connected to pixel }(i, j) \\
& \sqrt{(m-i)^{2}+(n-j)^{2}} \leq R
\end{aligned}
$$

These tests are performed for all pixels (e.g., $(m, n)$ for all $m$, and $n)$ to obtain the final homogeneous region for the seed pixel $(i, j)$. The statistical similarity criteria, the connectivity, and spatial distance bound, expressed in (4), (5), (6), respectively, are used to control the shape and size of growing region, and hence tradeoff between the speckle suppression and signal preservation. The spatial distance bound, $R$, is a consequence of the locality, and hence depends on the kernel size used for computation of statistics. In ASSF, for each seed pixel the $R$ is chosen in such a way that the total number of pixels in the grown region does not exceed $W^{2}$. Test of the connectivity is easily realized in the growing by following the neighboring pixels of seed pixel and/or pixels included in the region.

The statistical similarity bound, $\Delta \alpha($.$) , is chosen as a function of \alpha_{i, j}$ as:

$$
\Delta \alpha\left(\alpha_{i, j}\right)=a+b \exp \left\{-c \alpha_{i, j}\right\}
$$

where $a, b$, and $c$ are coefficients depending on fully developed statistics and level of desired smoothing. The coefficient $a$ is a biasing factor which controls smoothing employed both on speckle and signal, e.g., larger values of $a$ results in higher smoothing of both speckle and edges. The coefficient $b$ is a scaling factor used for adjusting the magnitude of statistical bound. Larger values of $b$ increases smoothing level as far as $c$ allows. The third coefficient, $c$, controls the value and sharpness of statistical threshold, and hence directly depends on $\alpha$ of fully developed speckle. The pixel with statistics below this threshold is forced to be smoothed higher and vice verse. Since speckle statistics dominantly depends on the scanner specifications, the coefficients of (7) can be determined at once, and stored in a look-up table. The $\Delta \alpha(\alpha)$ for normalized $\alpha$ is given in Fig. 2.

Following the region growing, the standard mean filtering is performed over the obtained region as the filter kernel, and the filtering result is assigned to the image value of the associated seed pixel. Additionally, after the filtering of whole image, the neighbor regions with the similar intensity values are merged to prevent the high frequency artifacts iue to the filtering with arbitrary shaped and sized kernels.

Overall procedure of ASSF can be outlined as follows:

- make the table for growing criteria (Eq. 7). 
- compute local statistics for all pixels (Eq. 3).

- for all pixels:

- grow homogeneous region (Eq. 4).

- take mean of pixels in the region (Eq. 1).

- output mean

- Merge neighboring regions with means close to each other by one gray level.

\section{RESULTS AND COMPARISON}

The image used in this study (see Fig. 3.a) is a subsection of a 90-degrees phased array sector image. The image is reconstructed by digital processing of radio frequency data acquired from an ultrasound standard tissue mimicking resolution phantom with a $3.3 \mathrm{MHz}, 64$-element transducer array. The image has a $60 \mathrm{~dB}$ dynamic range resulting from the logarithmic compression of envelope detected echo. It has a size of $256 \times 256$ pixels, and a pixel resolution of $0.23 \mathrm{~mm}$.
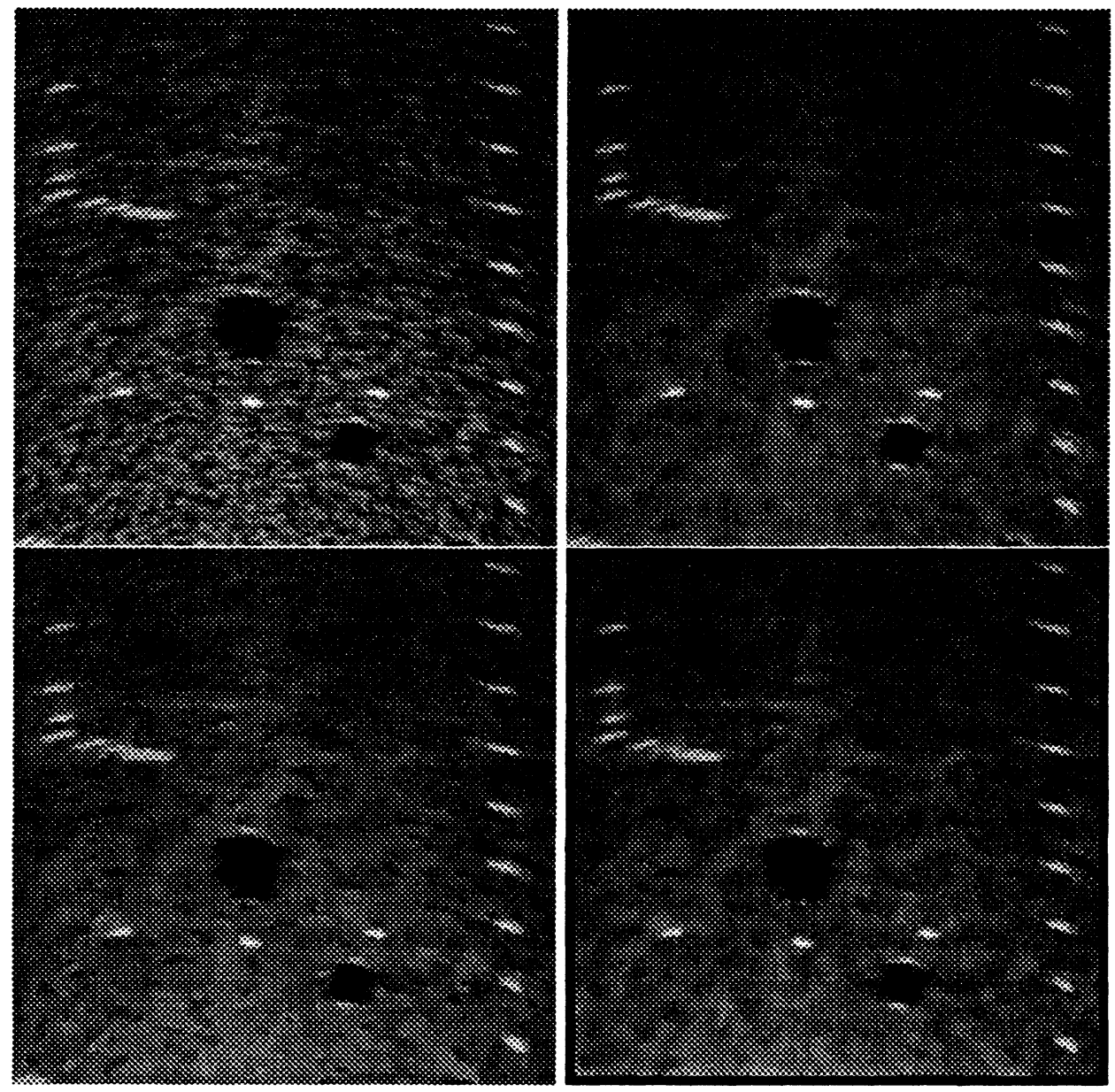

Fig. 3: The B-scan image (a) original and filtered images by (b) ASSF, (c) HGRMF, (d) AWMF. 


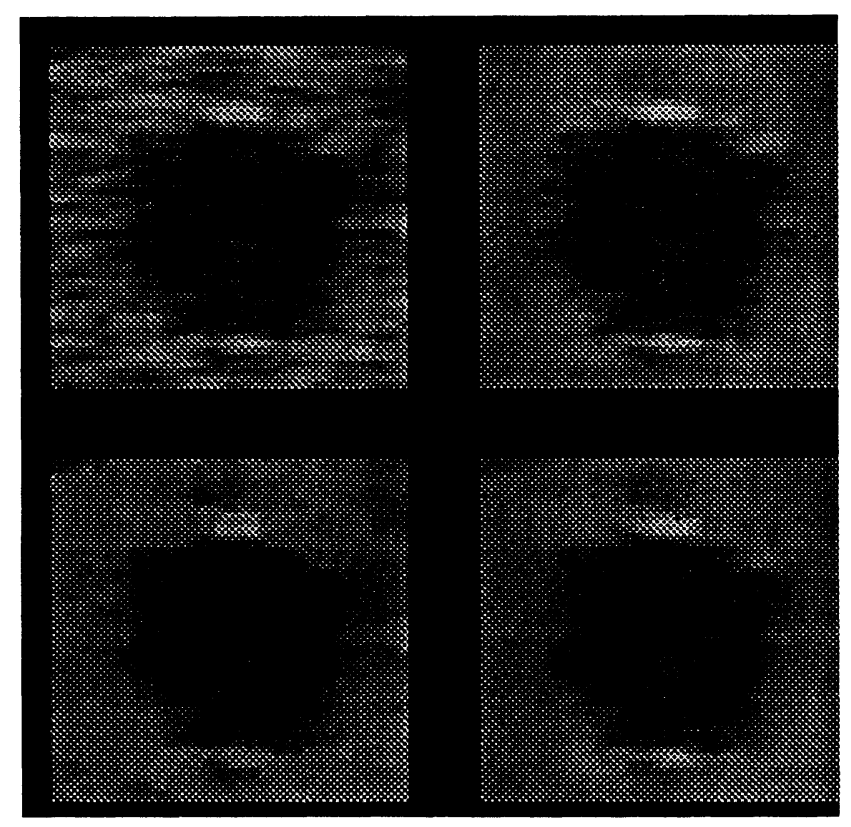

Fig. 4: A section of the image enlarged by three times using zero-order interpolation. (a) Original and output of (b) ASSF, (c) HRGMF, and (d) AWMF.

Performance of ASSF is tested on the phantom image. Fig. 3.a and $b$ show the original and filtered images. It is observed that ASSF effectively filters out the speckle while it preserves edges and subtle gray level changes. This is clearly observed from the enlarged section of the image given in Fig. 4. Fig. 4 depicts that the edges and textural content of a resolved structure is preserved while the speckle region surrounding the object is smoothed.

Performance of ASSF is compared with two recently reported filters employing image local statistics in filter adaptation. The filter proposed in [11] is an adaptive weighted median filter (AWMF) whereas the other proposed in [12] is a homogeneous region growing mean filter (HRGMF). In AWMF, median filtering is performed on a fixed running window with weight coefficients adjusted according to the image local statistics. In HRGMF, the filtering kernels are obtained through a procedure like split and merge used in region growing based segmentation. The kernel shape is restricted to be rectangular in HRGMF.

The filter parameters for each technique are found experimentally and turned out to be the best choice. The filtered inages and the normalized variances along row 153 are shown in Fig. 3 and Fig. 5, respectively. The figures indicate that performance of the filters is comparable. They smooth speckle effectively on homogeneous regions. However, edge preservation capability of ASSF is superior to other two filters (see Fig. 4). For the enlarged image section shown in Fig. 4, HRGMF and AWMF smooth the textural content of the object whereas ASSF preserves it. Such details are essential for clinical examination (Fig. 4.b- 4.d).

As far as computation time is concerned the most efficient one is AWMF. The proposed method and HGRMF has comparable run times. However, this is not critical in off-line applications. 


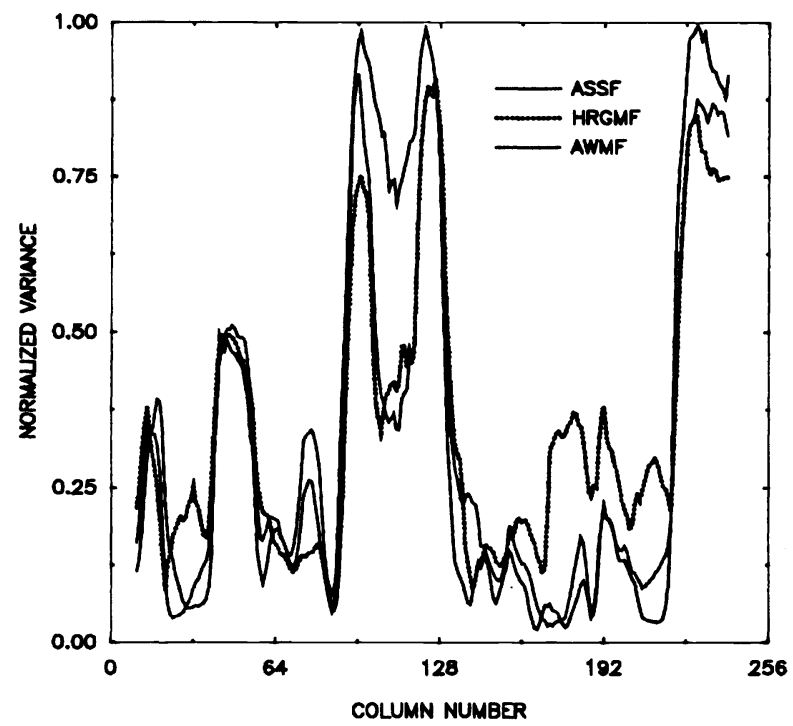

Fig. 5: Distributions of the variance along row 153 the filtered images where each is normalized by the variance along row 153 of the original image.

\section{CONCLUSION}

An adaptive filter for smoothing signal dependent noise is presented. Its application for suppression of speckle on ultrasound images is discussed. Smoothing is achieved by employing mean filtering on kernels obtained by local statistics based region growing procedure. The filter makes use of local image content thus eliminates noise effectively while preserving resolvable details. The tradeoff between smoothing and signal preservation is controllable by the filter adaptation parameters. The proposed filtering technique can also be used for reduction of signal dependent noise in images other than ultrasound B-scans, that show noise characteristics similar to speckle noise.

\section{REFERENCES}

[1] I. Pitas and A. N. Venetsanopoulos, "Nonlinear mean filters in image processing," IEEE Trans. Acoust. Speech Signal Process., vol. ASSP-34, pp. 573-584, June 1986.

[2] R. Bernstein, "Adaptive nonlinear filters for simultaneous removal of different kinds of noise in images," IEEE Trans. Circuits and Systems, vol. CAS-34, pp. 1275-1291, Nov. 1987.

[3] J. S. Lee, "Digital image enhancement and noise filtering by use of local statistics," IEEE Trans. Pattern Anal. Machine Intel., PAMI-2(2), 165-168, 1980.

[4] X. Z. Sun and A. N. Venetsanopoulos, "Adaptive schemes for noise filtering and edge detection by use of local statistics," IEEE Trans. Circuits and Systems, vol. CAS-35, pp. 57-69, Jan. 1988.

[5] J. W. Goodman, "Some fundamental properties of laser speckle," J. Opt. Soc. Amer., vol. 66, pp. 1145-1150, Nov. 1976. 
[6] C. B. Burckhordt, "Speckle in ultrasound B-mode scans," IEEE Trans. Sonic. Ultrason., vol. SU-25, pp. 1-6, Jan. 1978

[7] J. G. Abbott and F. L. Thurnstone, "Acoustic speckle:Theory and experimental analysis," Ultrason. Imag. , vol. 1, pp. 303-324, 1979.

[8] E. R. Ritenour, T. R. Nelson, and U. Raff, "Applications of the median filter to digital radiographic images," Proc. of IEEE Int. Conf. Acoust. Speech Signal Process., San Diego, CA, pp. 23.1.1-23.1.4, 1984.

[9] A. C. Bovik, T. S. Huang, and D. C. Munson, "A generalization of median filtering using linear combination of order statistics," IEEE Trans. Acoust. Speech Sig. Process., ASSP-31, pp. 1342-1349, 1983.

[10] D. T. Kuan, A. A. Sawchuk, T. C. Strand, and P. Chavel, "Adaptive noise smoothing filter for images with signal-dependent noise," IEEE Trans. Pattern Anal. Machine Intel., PAMI-7(2), 165-177, 1987.

[11] T. Loupas, W. N. McDicken and P. L. Allan, "An adaptive weighted median filter for speckle suppression in medical ultrasonic images," IEEE Trans. Circuits and Systems, vol. 36, no. 1, pp. 129-135, Jan. 1989

[12] J. I. Koo, S. B. Park, "Speckle reduction with edge preservation in medical ultrasonic images using a homogeneous region growing mean filter," Ultrasonic Imaging, 13, pp. 211-237, 1991.

[13] W. J. Song, W. A. Pearlman, "Edge preserving noise filtering based on adaptive windowing," IEEE Trans. Circuit and Systems, 35, pp. 1048-1055, 1988.

[14] J. C. Bamber and C. Daft, "Adaptive filtering for reduction of speckle in ultrasonic pulse-echo images," Ultrasonics, pp. 41-44, Jan. 1986.

[15] Y. Wu, H. Maitre, "Smoothing speckled synthetic aperture radar images by using maximum homogeneous region filters," Optical Engineering, vol. 31, no. 8, pp. 1785-1792, August 1992.

[16] F. M. Wohl, Digital Image Signal Processing, Artech House, Boston-London, 1987.

[17] S. L. Horowitz and T. Pavlidis, "Picture segmentation by a directed split and merge procedure," Proc. 2nd Int. Joint Conf. on Pattern Recognition, IEEE, New York, 1974.

[18] S. W. Zucker, "Region growing: childhood and adolescence," Comput. Graphics Image Process., vol. 5, pp. 382$399,1976$. 